\title{
The correlation of HPV16 and HPV18 with local vaginal immunity after the treatment of cervical intraepithelial neoplasia
}

\author{
Lin Mu", Jing-Rui Miao", Jing-Hui Song \\ Department of Obstetrics and Gynecology, Affiliated Hospital of Inner Mongolia Medical University, Inner Mongolia, China \\ Contributions: (I) Conception and design: L Mu, JR Miao; (II) Administrative support: JR Miao, JH Song; (III) Provision of study materials or \\ patients: JR Miao, JH Song; (IV) Collection and assembly of data: L Mu; (V) Data analysis and interpretation: JR Miao; (VI) Manuscript writing: All \\ authors; (VII) Final approval of manuscript: All authors. \\ \#These authors contributed equally to this work. \\ Correspondence to: Jing-Hui Song. Department of Obstetrics and Gynecology, Affiliated Hospital of Inner Mongolia Medical University, No. 1 of \\ North Tongdao street, Huimin District, Inner Mongolia 100059, China. Email: jinghuisong277@163.com.
}

Background: This study aimed to investigate the correlation between the level of HPV16 and HPV18 in patients with cervical lesions and the local vaginal immunity after receiving treatment with different degrees of cervical intraepithelial neoplasia (CIN) and cervical squamous cell carcinoma (CSCC).

Methods: One hundred and thirty-six patients with LSIL (grade 1 CIN or CIN 1), 263 patients with HSIL (grade 2 and 3 CIN or CIN 2 and 3), and 33 patients with CSCC pathologically confirmed between November 2012 and September 2016 were assigned to the test group and 100 healthy women in the same period were assigned to the control group. ELISA was used to determine the levels of SIgA, IgG, IL-2, and IL-10 and the IL-2/IL-10 ratio in vaginal lavage fluid in the test group (before treatment and 3, 6, and 12 months after treatment) and the control group, respectively. Gene chip technology was adopted to test the HPV infection in the test group (before treatment and 3, 6, and 12 months after treatment) and the control group, respectively.

Results: The results showed that the levels of SIgA, IgG, and IL-10 were positively correlated with the degree of cervical lesions and IL-2/IL-10 was negatively correlated with the degree in all patients before treatment. The levels of SIgA, IgG, and IL-10 were positively correlated with the degree of cervical lesions and IL-2/IL-10 was negatively correlated with the degree in HPV16 and HPV18-infected patients before receiving the treatment.

Conclusions: The recovery after the treatment of HPV infection is time-dependent and the immune system of patients with CIN starts to recover in the 6th month after treatment, early intervention is not recommended. However, the immune system is approximately in a normal sate at 12 months after treatment, at which time the efficacy can be evaluated, and clinical intervention can be initiated if necessary. The level of local immune factors in the vagina can be monitored to determine the progression and prognosis of patients' cervical lesions.

Keywords: Cervical intraepithelial neoplasia (CIN); cervical squamous cell carcinoma; HPV 16; HPV 18; vaginal local immune factor

Submitted Dec 25, 2019. Accepted for publication Jun 05, 2020.

doi: $10.21037 /$ tcr-19-2955

View this article at: http://dx.doi.org/10.21037/tcr-19-2955 


\section{Introduction}

Cervical intraepithelial neoplasia (CIN) is an infectious disease caused by high-risk human papillomavirus (HRHPV) (1-3). The available studies suggest that $75 \%$ of sexually active women will have at least one HR-HPV infection in their lifetime, of which $90 \%$ are transient and only $10 \%$ of patients develop a persistent infection. Approximately $5 \%$ of persistent infections will also progress to CIN (4-7).

The mucosal immune was the first immune barrier against infection in the body. SIgA formation is a hallmark of the mucosal immune response and was directly related to local immunity. B cells in local mucosal tissues can also produce IgG and IgM independently, which were one of the major antibodies involved in the body's secondary immune response. In addition to humoral immunity, mucosal immunity also produces mucosal cytotoxic $\mathrm{T}$ lymphocyte responses (CTLs). Mature T lymphocytes express CD4+ and CD8+ cells. CD4+ cells, including subsets of Th1, Th2, Th17, and Treg and acting as the primary cells involved in tumor immunity, assist and boost other immune cells in killing tumor cells by secreting cytokines.

Will local vaginal immunity affect HPV infection, pathogenicity, and the outcome when the cervix is exposed in the vagina? To answer this question, we conducted this trial to investigate the correlation between the pathogenicity and the outcome of HPV16 and HPV18 in patients with CIN and CSCC and the local vaginal immunity by determining the levels of Secretory immunoglobulin A (SIgA), Immunoglobulin G (IgG), Interleukin-2, 10 (IL2 , IL-10), and IL-2/IL-10 ratio in the patients' vaginal lavage fluid before treatment and 3, 6, and 12 months after treatment in HPV16 and HPV18-positive patients with different degrees of CIN and CSCC.

\section{Methods}

The study was conducted in accordance with the Declaration of Helsinki (as revised in 2013). The study was approved by institution ethics board of Affiliated Hospital of Inner Mongolia Medical University (No.: 2019029) and informed consent was taken from all the patients.

\section{General data}

The patients with LSIL (grade 1 CIN or CIN 1) and patients with HSIL (grade 2 and 3 CIN or CIN 2 and 3) were included in this study between November 2012 and September 2015 at the Department of Obstetrics \& Gynecology in the Affiliated Hospital of Inner Mongolia Medical University. Patients with LSIL received medication (including a Baofukang suppository and recombinant human interferon) or physiotherapy, with the course of treatment being 3 months. HSIL patients with CIN 2 received the loop electrosurgical excision procedure (LEEP), patients with CIN 3 received cold knife conization (CKC), and patients with CSCC received epifascial panhysterectomy or radical hysterectomy + pelvic lymphadenectomy. No other assisted therapies were required after the operation was completed. Another 100 healthy women without cervical lesions in the same period were enrolled in the control group. All the enrolled patients completed the questionnaire on basic information and signed informed consent. The study complied with the medical ethics of clinical trials.

\section{Inclusion and exclusion criteria}

Inclusion criteria: (I) patients with no systemic antibiotic application within 2 weeks; (II) patients with no sex hormone application within 3 months; (III) patients that were not in the vaginal bleeding period; (IV) patients that did not receive any vaginal medication within 48 hours before the visit to the hospital; (V) patients with no sexual activity, tub bath, vaginal washing, and operation within 24 hours before the visit to the hospital; (VI) patients with a negative result of vaginal smear testing before the sampling was completed; (VII) patients that did not receive any radiotherapy or chemotherapy before the sampling was completed.

Exclusion criteria: (I) pregnant or lactating women; (II) patients with severe heart, lung, liver, kidney, and hematopoietic system diseases and psychosis; (III) patients with immunocompromise (e.g., patients with malignant tumors, immune diseases or taking immunosuppressor); (IV) patients who had deep muscle invasion; (V) patients who had intravascular cancer embolus; (VI) patients who had lymphatic metastasis; (VII) patients who had a positive margin or the margin was $<2 \mathrm{~cm}$ from the tumor.

\section{Method of test group follow-up time}

The vaginal lavage fluid and cervical secretion were collected on the first day when the patients were admitted to the test group and control group before treatment. Vaginal lavage fluid and cervical secretion were collected in 
the test group (before treatment and 3, 6, and 12 months after treatment) and the control group to investigate the level of HPV16 and HPV18 after the different durations of treatment. Enzyme-linked immuno sorbent assay (ELISA) was used to determine the levels of SIgA, IgG, IL-2, and IL-10 in the patients' vaginal lavage fluid in the test group (before treatment and 3, 6, and 12 months after treatment) and the control group, respectively. Gene chip technology was adopted to test the HPV in the patients' cervical secretion. The follow-up on all the enrolled patients was under the supervision of a clinician in our hospital. All patients were reminded beforehand through telephonic communication of the follow-up visit in our hospital. Patients that had difficulty in attending the follow-up were provided with assistance. The enrolled patients who had been re-examined in other hospitals were suggested to register in our hospital and undergo the collection of vaginal lavage fluid and cervical secretion. All patients were suggested to attend the follow-up as scheduled. Drop-out patients were excluded, and new patients were enrolled in the study.

\section{Specimen treatment and requirements}

All patients were sexually active and not pregnant; they presented no vaginal bleeding, acute reproductive organ inflammation, systemic antibiotic application within 2 weeks, sex hormone application within 3 months, vaginal lavage drug administration within 1 week and they also had no sexual activity, tub bath, vaginal washing or operation within 24 hours before the visit to the hospital. The patients also had negative results from the vaginal smear test and no radiotherapy or chemotherapy before sampling. Sampling was performed at 3-7 days after the patients' experienced menstruation before treatment and 3, 6, and 12 months after treatment in the test group, respectively. Only one sampling method was required in the control group.

\section{The collection of vaginal lavage fluid}

The unlubricated vaginal speculum was used to expose the inside of the vagina and cervix and then $0.9 \%$ of Sodium Chloride Solution was used to flush the upper 1/3 of the vaginal wall and the posterior fornix. The vaginal lavage fluid was collected in a tube and kept at $-20{ }^{\circ} \mathrm{C}$, followed by 20 minutes of centrifuging (2,000-3,000 rpm) after dissolution at room temperature. Thereafter, the supernatant was collected and subject to the immune factor (SIgA, IgG, IL-2, IL-10) test with ELISA.

\section{The HPV test}

A specifically designed sampling brush for HPV was inserted into the cervix and rotated clockwise for 3-5 full rotations. The brush head was placed in a sample tube filling with the cell preservative solution for the subsequent submission of the test. Gene chip technology was used to test the HPV infection in patients.

\section{ELISA}

ELISA was applied to determine the levels of humoral immune factors, such as SIgA, IgG and cellular immune factors, such as IL-2 and IL-10 in the vaginal lavage fluid. Principle: A double-antibody sandwich ELISA was used to determine the levels of SIgA, IgG, IL-2, and IL-10 in the sample. Purified anti-SIgA, anti-IgG, anti-IL-2, and antiIL-10 antibodies were used to coat a microtiter plate to make solid-phase antibodies. SIgA, IgG, IL-2, and IL-10 were added to all wells that were coated with monoclonal antibody successively. Then, the antibodies in the wells were conjugated with $\mathrm{H}$ horseradish peroxidase (HRP)-labeled goat anti-human antibody to form the antibody-antigenenzyme-labeled antibody complex. After the wells were thoroughly washed, substrate TMB was used for the color development; TMB yielded a blue color upon reaction with the HRP and then turned yellow after the addition of the acid solution. A microplate reader was used to determine the absorbance (OD value) at a $450 \mathrm{~nm}$ wavelength. A standard curve was constructed to calculate the levels of SIgA, IgG, IL-2, and IL-10 in the sample tested.

\section{Reagents and equipment}

RDelisa SIgA, IgG, IL-2, IL-10 ELISA kit: Shanghai Jinma Biological Technology Inc. The composition of the ELISA kit: Assay plate: one (96-well). Standard substance: $0.5 \mathrm{~mL} \times 1$ vial. Standard substance diluent: $1.5 \mathrm{~mL} \times 1$ vial. Enzyme conjugate: $6 \mathrm{~mL} \times 1$ vial. Sample diluent: $6 \mathrm{~mL} \times$ 1 vial. Chromogenic reagent $\mathrm{A}$ : $6 \mathrm{~mL} \times 1$ vial. Chromogenic reagent B: $6 \mathrm{~mL} \times 1$ vial. Stop solution: $6 \mathrm{~mL} \times 1$ vial. Wash concentrate: $(20 \mathrm{~mL} \times 30$-fold $) \times 1$ bottle. Other reagents: sterile $0.9 \%$ of sodium chloride injection, distilled water. The main test instruments and equipment are listed in Table 1.

\section{Procedures}

(I) Dilution of the standard substance: We added the standard substance to 10 wells of the coated microplate and then diluted the standard in series as described in the 
Table 1 The main test instruments and equipment

\begin{tabular}{lcl}
\hline Name & Model & Manufacturer \\
\hline Microplate reader & Model RT-2100C & Rayto Life and Analytical Sciences Co., Ltd., Shenzhen \\
Automatic microplate washer & Model RT-3000 & Rayto Life and Analytical Sciences Co., Ltd., Shenzhen \\
Refrigerator & Model YC-280L & Zhongke Meiling Cryogenics Co., Ltd. \\
Centrifuge & Model TD5A-WS & Changsha Xiangyi Centrifuge Instrument Co., Ltd. \\
Electric incubator & Model HPX-9082ME & Medical Equipment Factory, Shanghai Boxun Industry \& Commerce \\
& & Co., Ltd. \\
Micropipette & $1,000 \mu \mathrm{L}, 200 \mu \mathrm{L}$ & Purui \\
EP tube & $1.5 \mathrm{~mL}$ & \\
Tip & $1,000 \mu \mathrm{L}, 200 \mu \mathrm{L}$ & \\
Centrifugal tube & $5 \mathrm{~mL}$ & \\
\hline
\end{tabular}

instructions. After the dilution, the loading amount per well was $50 \mu \mathrm{L}$ and the concentrations were $180,120,60$, 30, and $15 \mathrm{U} / \mathrm{mL}$, respectively (2). Sample loading: Blank wells and sample wells were designed. We then added a $40 \mu \mathrm{L}$ sample diluent to each sample well of the coated microplate, followed by the addition of a $10 \mu \mathrm{L}$ sample to be tested (thus, the sample was finally diluted 5 -fold) (3). Incubation: we sealed the plate with the microplate sealer and then incubated it at $37^{\circ} \mathrm{C}$ for 30 minutes (4). Solution preparation: we diluted the 30 -fold (20-fold for $48 \mathrm{~T}$ ) wash concentrate 30 -fold ( 20 -fold for $48 \mathrm{~T}$ ) with distilled water for subsequent use (5). Washing: we removed the sealer and discarded the liquid, dried the microplate, filled each well with the washing solution, let it stand for 30 seconds, and then discarded the washing solution. These procedures were repeated 5 times and then we dried the plate (6). Enzyme loading: we added $50 \mu \mathrm{L}$ of enzyme conjugate to each well, excluding the blank wells (7). Incubation: the same as step 3 (8). Washing: the same as step 5 (9). Color development: we added $50 \mu \mathrm{L}$ of the chromogenic reagent $\mathrm{A}$ to each well, followed by $50 \mu \mathrm{L}$ of the chromogenic reagent $\mathrm{B}$. We then mixed the plate gently to mix the reagents well and developed it for 15 minutes away from light at $37{ }^{\circ} \mathrm{C}(10)$. Termination: we added $50 \mu \mathrm{L}$ of the stop solution to each well to stop the reaction (the color turns from blue to yellow immediately) (11). Determination: a zero setting was performed based on the well and determined the absorbance (OD value) of each well at a $450 \mathrm{~nm}$ wavelength sequentially. The determination should be conducted within 15 minutes upon the addition of the stop solution.

\section{Calculation}

We constructed a standard curve by plotting the concentration of the standard on the $\mathrm{X}$-axis and the $\mathrm{OD}$ values on the Y-axis. We then identified the corresponding concentration of the sample to the OD value and then multiplied the concentration by the dilution factor or formulated a linear regression equation of the standard curve with the concentration and OD value of the standard substance and then substituted the OD value of the sample into the equation to calculate the sample concentration. Finally, we multiplied the dilution factor to get the actual concentration of the sample.

\section{Gene chip technology}

Gene chip technology was used to test the HPV infection. We suspended the clinical sample for 10 seconds and added $500 \mu \mathrm{L}$ of the sample to a sample tube. It was then centrifuged for 1 minute at 14,000 rpm and the supernatant was discarded. We added solutions I, II, and III successively, as described in the instructions. $1 \mu \mathrm{L}$ of the dissolved sample was then stored in a refrigerator at $-20{ }^{\circ} \mathrm{C}$ for subsequent use.

\section{PCR}

Reagent: Hybribio HPV genotyping assay kit-PCR amplification kit. We prepared the PCR as per the steps in the IFU. The PCR primers sequences were: HPV-F: 5'-CGTCCAAGAGGATACGATC-3', HPV-R: 5'-GCACAGGGACATAATAATGG-3', GAPDH-F: 5 ' - CAACTCCCTCAAGATTGTCAGCAA-3 ', 
GAPDH-R: 5'-GGCATGG ACTGTGGTCATGA-3' . PCR amplification: We put the PCR tube in the PCR machine in the PCR amplification area to initiate the PCR as the following procedures:

$95^{\circ} \mathrm{C} 9$ minutes $\longleftarrow$
$95^{\circ} \mathrm{C} 20$ seconds
$55^{\circ} \mathrm{C} 30$ seconds $\longleftarrow$
$72{ }^{\circ} \mathrm{C} 30$ seconds
$72{ }^{\circ} \mathrm{C} 5$ minutes
$4{ }^{\circ} \mathrm{C}$ Incubation

\section{SOP for the flow-through hybridization}

SOP for the flow-through hybridization: HPV genotyping assay kit-hybridization kit. Procedures: (I) we took the $45^{\circ} \mathrm{C}$ hybridization solution out of the preheating water bath and then added $1 \mathrm{~mL}$ of the $45{ }^{\circ} \mathrm{C}$ hybridization solution to the hybridization that was covered well with the hybridization film and incubated it for 2 minutes; (II) we took the PCR product to be hybridized from the refrigerator. After the dissolution, the product was centrifuged in an electric centrifuge and then put it in the PCR machine for a 5 -minute degeneration at $95^{\circ} \mathrm{C}$. We kept the degenerated product in ice water for at least 2 minutes; (III) we took the $1.5 \mathrm{~mL}$ centrifugal tubes of the same number as the samples and marked them with sample numbers; (IV) we opened the pump, discharged the pre-hybridized solution, and then closed the pump; (V) we took the $45^{\circ} \mathrm{C}$ hybridization solution out of the preheating water bath. $0.5 \mathrm{~mL}$ of the solution was put in the tube of each $1.5 \mathrm{~mL}$ centrifugal tube on the tube rack quickly; (VI) we took the PCR products out of the ice-water bath in the order of the sample numbers. $20 \mu \mathrm{L}$ of the PCR product was put in the tube of each $1.5 \mathrm{~mL}$ centrifugal tube. We mixed them well using the tip and then added the mixture to the hybridization well in the compartment. We returned the $1.5 \mathrm{~mL}$ tubes to the tube rack (in the same order as the placement of the samples in the compartment) and repeated this step until all samples were added; (VII) we covered the instrument and allowed for hybridization for 10 minutes at $45^{\circ} \mathrm{C}$ and put the hybridization solution back in the water bath; (VIII) we recorded the sample numbers and loading locations on a record book referencing the numbers of the $1.5 \mathrm{~mL}$ centrifugal tubes on the rack; (IX) after 10 minutes of hybridization, we opened the pump for flow-through hybridization, drained the solution on the hybridization film, and then closed the pump; (X) we then took the $45^{\circ} \mathrm{C}$ hybridization solution out of the preheating water bath. $0.8 \mathrm{~mL}$ of the solution was added to flush the film. We then opened the pump to drain the solution on the film and then closed the pump. This step was repeated 3 times.

\section{Color development}

(I) We set the hybridization instrument temperature to $25^{\circ} \mathrm{C}$; (II) added $0.5 \mathrm{~mL}$ of the blocking buffer to seal the film. When the temperature dropped to $30^{\circ} \mathrm{C}$, we opened the pump to drain all the blocking buffer and then closed the pump; (III) we added another $0.5 \mathrm{~mL}$ of the blocking buffer to seal the film, covered the instrument, and opened the pump after 5 minutes and then drained the $0.5 \mathrm{~mL}$ buffer and closed the pump; (IV) when the temperature dropped to $25^{\circ} \mathrm{C}\left( \pm 1^{\circ} \mathrm{C}\right)$, we added $0.5 \mathrm{~mL}$ of the enzyme conjugate and then covered the instrument and incubated for 3.5 minutes. The pump was then opened, we drained the $0.5 \mathrm{~mL}$ enzyme conjugate, and then closed the pump; (V) we set the hybridization instrument temperature to $36^{\circ} \mathrm{C}$; (VI) we added $0.8 \mathrm{~mL}$ of the solution A to flush the film, opened the pump to drain the solution on the film, and then closed the pump. This step was repeated 4 times; (VII) when the temperature increased to $36^{\circ} \mathrm{C}\left( \pm 1.0{ }^{\circ} \mathrm{C}\right)$, we shook the brown vial of the NBT/BCIP solution, added $0.5 \mathrm{~mL}$ of the NBT/BCIP solution, and covered the plate to allow for the color development for approximately 3-5 minutes; (VIII) we then opened the pump to pump out all the NBT/BCIP solution; (IX) $0.8 \mathrm{~mL}$ of solution B was then added to flush the film and then we opened the pump to drain the solution on the film and closed the pump. We repeated this step three times; $(\mathrm{X}) 1 \mathrm{~mL}$ of distilled water was added to flush the film. We then opened the pump to drain the solution on the film and then closed the pump. We opened the Snap-On cap, removed the compartment, took out the hybridization film with forceps and put it on an absorbent paper to dry. Then, we marked the sample number at the bottom left of the film with a pencil in the order of the placement of the samples recorded on the record book.

\section{Data analysis}

All data were collected and analyzed by the Statistics Office at the Tianjin Medical University. Analytical method: In the present study, we described all the data with a statistical method. Quantitative data were expressed as $\bar{x} \pm s$ and qualitative data was expressed with absolute and relative indexes, such as the number of cases and constituent ratio. SAS 9.2 was used for the statistical analysis. The statistical results were presented in statistical statements. A two-tailed 
Table 2 Expression of SIgA, IgG, IL-2 and IL-10 and IL-2/IL-10 ratio before treatment in test groups and the control group

\begin{tabular}{|c|c|c|c|c|c|c|}
\hline Group & Number & SlgA & $\lg G^{*}$ & IL-2* & IL-10* & IL-2/IL-10* \\
\hline LSIL & 136 & $1.090(1.347)$ & $3.894(5.94)$ & 63.544 (21.894) & 13.411 (6.909) & 9.967 (14.876) \\
\hline HSIL & 263 & $1.296(1.174)$ & $6.617(8.366)$ & 45.095 (17.068) & $18.443(8.863)$ & $4.418(6.242)$ \\
\hline $\operatorname{cscc}$ & 33 & $1.421(1.009)$ & 6.776 (9.587) & $80.863(72.047)$ & $45.882(16.573)$ & 2.357 (2.429) \\
\hline
\end{tabular}

${ }^{*}$, the difference between two groups is statistically significant.

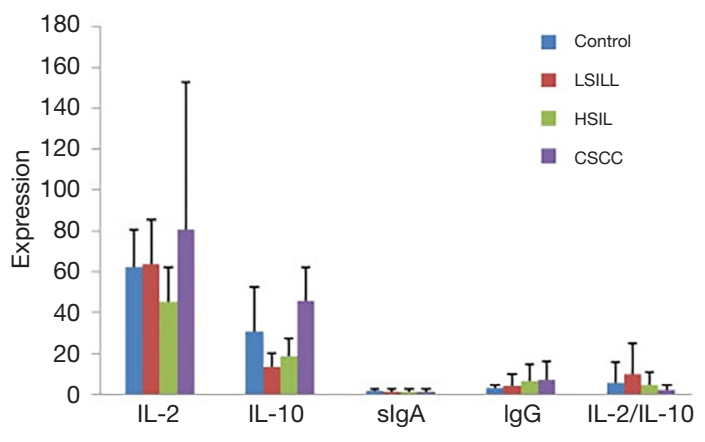

Figure 1 The Expression of SIgA, IgG, IL-2, and IL-10 and the IL-2/IL-10 Ratio Before Treatment in the Test Groups and the Control Group.

test was used for all statistical tests. When $\mathrm{P} \leq 0.05$, the difference between the groups was considered statistically significant. One-way ANOVA and two-way ANOVA were used for single-factor multilevel quantitative data and multi-factor quantitative data, respectively. A chi-square contingency table was used for single-factor qualitative data analysis and a CMH chi-square test was used for highdimensional contingency table data.

\section{Results}

\section{The general characteristics}

In the test group, there were 136 patients with LSIL (grade 1 CIN or CIN 1), including 56 HPV16 and HPV18positive patients (mean age $41.81 \pm 7.97$ years) and 263 patients with HSIL (grade 2 and 3 CIN or CIN 2 and 3), including 112 HPV16 and HPV18-positive patients (mean age $42.22 \pm 9.13$ years). Thirty-three patients with CSCC were at stage IA1-IIA1, including 28 HPV16 and HPV18positive patients (mean age $50.70 \pm 11.06$ years). In the control group, there were 100 healthy women (mean age
$42.22 \pm 8.76$ years) without cervical lesions, including 74 $\mathrm{HPV}$-negative women, $22 \mathrm{HR}-\mathrm{HPV}$-infected women, 1 multiple HPV infected-woman, and 3 low-risk HPVinfected women.

\section{The level of SIgA, IgG, IL-2, and IL-10}

The expression results of the SIgA, IgG, IL-2, and IL10 and the IL-2/IL-10 ratio before treatment in the test groups and the control group are listed in Table 2. The ANOVA expression in the different immune factors before the treatment of the different lesion groups showed a statistically significant difference $(\mathrm{P}<0.0001)$. The comparison between any two groups showed a lower SIgA value in the test group than in the control group. However, as the lesion grade increased, this factor was expressed at a higher level. IL-2, IL-10, and IgG were expressed at the highest level in the CSCC group and the IL-2/IL-10 ratio presented the highest in the LSIL group. With the increase of the lesion grade, the patient's immunity was highly expressed. The levels of SIgA, IgG, and IL-10 were positively correlated with the degree of the cervical lesions and IL-2/IL-10 was negatively correlated with the degree (Figure 1).

The expression results of the SIgA, IgG, IL-2, and IL-10 and the IL-2/IL-10 ratio before treatment in HPV16 and HPV18-positive patients in the test groups and the control group are listed in Table 3. The ANOVA expression in the different immune factors before the treatment in HPV16 and HPV18-infected patients in the different lesion groups showed a statistically significant difference $(\mathrm{P}<0.0001)$ on indexes other than the SIgA. The comparison between any two groups showed a lower expression of IL-2 in the HSIL group than in the other groups. The IL-10 was expressed at the highest level in the CSCC group. The levels of SIgA, IgG, and IL-10 were positively correlated with the 
Table 3 Expression of SIgA, IgG, IL-2 and IL-10 and IL-2/IL-10 ratio before treatment in HPV16- and HPV18-positive patients in test groups and the control group

\begin{tabular}{lclllll}
\hline Group & Number & \multicolumn{1}{c}{ SlgA } & \multicolumn{1}{c}{$\operatorname{lgG}$} & \multicolumn{1}{c}{ IL-2* } & IL-10* \\
\hline Control & 9 & $1.624(0.447)$ & $2.68(1.28)$ & $55.66(17.01)$ & $36.36(21.42)$ & $3.09(4.19)$ \\
LSIL & 56 & $1.06(1.36)$ & $2.43(3.17)$ & $62.5(25.44)$ & $13.45(6.59)$ & $9.78(15.01)$ \\
HSIL & 112 & $1.34(1.23)$ & $6.70(8.24)$ & $42.50(18.90)$ & $19.37(8.86)$ & $3.99(6.13)$ \\
CSCC & 28 & $1.52(1.052)$ & $6.85(10.13)$ & $83.65(77.06)$ & $47.59(15.85)$ & $2.27(2.47)$ \\
P & & 0.282 & 0.00035 & $<0.0001$ & $<0.0001$ & 0.00259 \\
\hline
\end{tabular}

*, the difference between two groups is statistically significant.

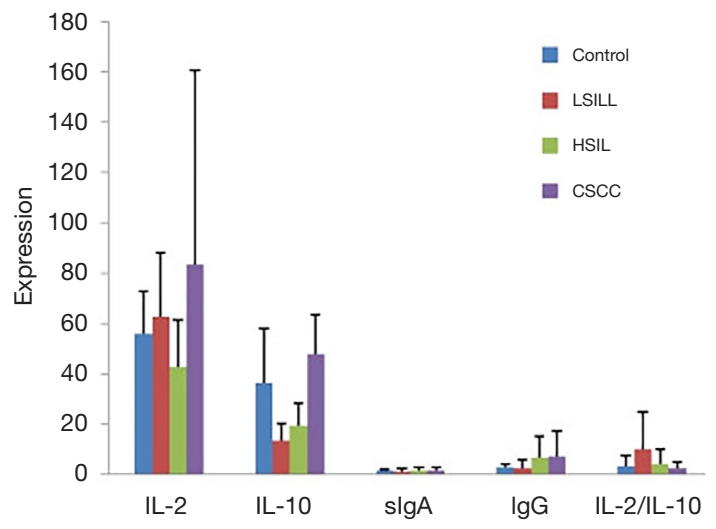

Figure 2 The Expression of SIgA, IgG, IL-2, and IL-10 and the IL-2/IL-10 Ratio After Treatment in HPV16 and HPV18-positive Patients in the Test Groups and the Control Group.

degree of the cervical lesions and IL-2/IL-10 was negatively correlated with the degree (Figure 2).

\section{The negative conversion rate of HPV before, 3, 6, and 12 months after the treatment of LSIL}

The negative conversion rate of HPV before, 3, 6, and 12 months after the treatment of LSIL are listed in Table 4. A chi-square test was used to analyze the negative conversion rate of HPV-infected patients with LSIL before and at different time points after treatment. The negative conversion rates of HPV infection varied with time $\left(\chi^{2}=160.7805, \mathrm{P}<0.0001\right)$. The rate increased with time and reached the highest $(92.94 \%)$ at the 12 th month after treatment. The negative conversion rate of HPV was positively correlated with the extension of the follow-up duration.

The correlation of HPV-negative conversion rates with immune factors before, 3, 6, and 12 months after the treatment in HPV16 and HPV18-positive patients with LSIL. The ANOVA of HPV infection and immune factor changed at different time points in HPV16 and HPV18infected patients with LSIL showed a statistically different expression of IL-10 $(\mathrm{F}=6.72, \mathrm{P}<0.0001)$, while the changes of IL-2, IL-2/IL-10, SIgA, and IgG were not statistically different $(\mathrm{P}>0.05)$. The expression amount of IL-10 increased with the treatment. The SIgA of patients with LSIL was negatively correlated with the follow-up duration and IL-10 level was positively correlated with the followup duration. IgG and IL-2 were expressed at a low level and IL-2/IL-10 was expressed at a high level 6 months after treatment.

\section{The negative conversion rate of HPV before, 3, 6, and 12 months after the treatment of HSIL}

The negative conversion rate of HPV before, 3, 6, and 12 months after the treatment of HSIL are listed in Table 5. A chi-square test of the recovery rate of HPVinfected patients with LSIL at different time points of treatment showed a statistically significant difference $\left(\chi^{2}=207.4137, \mathrm{P}<0.0001\right)$. The negative conversion rate reached the highest in the 12 th month after treatment. The negative conversion rate of HPV was positively correlated with the extension of the follow-up duration.

The Correlation of HPV Infection Outcome with Immune Factors before, 3, 6, and 12 months after the treatment in HPV16 and HPV18-infected patients with HSIL. The differences in IL-2, IgG, and IL-10 at different time points of treatment in various HPV infection cases were statistically significant ( $\mathrm{F}: 3.92, \mathrm{P}=0.0039 ; \mathrm{F}: 14.78$, $\mathrm{P}<0.0001 ; \mathrm{F}: 8.07, \mathrm{P}<0.0001)$, as compared with SIgA. $\mathrm{IgG}$ increased with treatment in HPV (positive) cases but decreased after 12 months of treatment compared with 
Table 4 Negative conversion rate of HPV before, 3, 6 and 12 months after treatment of LSIL (n, \%)

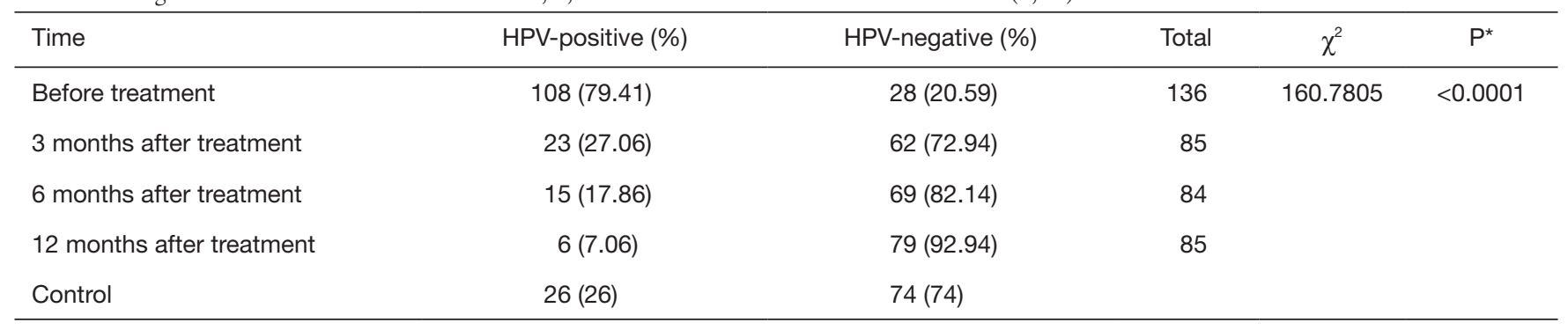

*, $P<0.001$ is considered statistically significant.

Table 5 Negative conversion rate of HPV before, 3, 6 and 12 months after treatment of HSIL (n, \%)

\begin{tabular}{lcccc}
\hline \multirow{2}{*}{ Group } & \multicolumn{2}{c}{ HPV (\%) } & Total & $\chi^{2}$ \\
\cline { 2 - 4 } & Negative & Positive & & 207.4137 \\
\hline Normal & $74[74]$ & $26[26]$ & 20.0001 & \\
Before treatment & $49(18.63)$ & $214(81.37)$ & 263 & \\
3 months after treatment & $115(66.47)$ & $58(33.53)$ & 173 \\
6 months after treatment & $110(69.62)$ & $48(30.38)$ & 158 \\
12 months after treatment & $111(77.62)$ & $32(22.38)$ & 143 \\
Total & 459 & 378 & 837 \\
\hline
\end{tabular}

*, $\mathrm{P}<0.001$ is considered statistically significant.

the control group. Both IL-2 and IL-10 decreased with treatment and reached the lowest at 6 months, followed by an upward trend. The SIgA and IgG levels were negatively correlated with the extension of the follow-up duration of the HSIL patients. IL-2 and IL-10 were expressed at a low level and IL-2/IL-10 was expressed at the lowest level 6 months after treatment.

\section{Discussion}

The outcomes of this study showed that the levels of SIgA, IgG, and IL-10 were positively correlated with the degree of the patients' cervical lesions and IL-2/IL-10 was negatively correlated with the degree in all patients before treatment. The levels of SIgA, IgG, and IL-10 were positively correlated with the degree of cervical lesions and IL-2/IL10 was negatively correlated with the degree in HPV16 and HPV18-infected patients before treatment. In the LSIL group, the negative conversion rate of HPV was positively correlated with the extension of the follow-up duration and the IL-10 level was positively correlated with the follow-up duration in the LSIL patients. IgG and IL-2 were expressed at a low level and IL-2/IL-10 was expressed at a high level 6 months after treatment. In the HSIL group, the negative conversion rate of HPV was positively correlated with the extension of the follow-up duration and the SIgA and IgG levels were negatively correlated with the extension of the follow-up duration in the HSIL patients. IL-2 and IL-10 were expressed at a low level and IL-2/IL-10 was expressed at the lowest level 6 months after treatment.

Similar to systemic immunity, local vaginal immunity consists of humoral immunity and cell-mediated immunity. The cervix is located inside the vagina, so local vaginal immunity will directly affect the cervix. When HPV infection occurs in the cervix, the anti-infection mechanism of vaginal immunity can resist the pathogen invasion (8). However, if any injury occurs in the systemic or local immunity, the HPV will damage the epithelial cells and evolve immune escape mechanisms while increasing the risk of cervical lesions and resulting in a vicious circle of the tumor and immune injury. HR-HPV persistent infection will last for more than 2 years before it progresses to CIN while HSIL can progress to cervical cancer in 10-20 years from the initial infection with $\operatorname{HPV}(9,10)$. 
The mucosal immune system consists of submucous lymphoid tissues lining the respiratory tract, gastrointestinal tract, and urogenital canal and those dispersed at some of the exocrine glands, including mucosa-associated lymphoid tissues $(11,12)$. It acts as the first immune barrier of the body against infection (13). SIgA formation is a hallmark of the mucosal immune response. It is a complex that incorporates dimeric IgA and J chain secretory component (SC) and an immune globulin on the mucosal surface with the largest secretion, accounting for $80 \%$ of the amount of mucosal immunoglobulin. It is not easily hydrolyzed by protease. The level of SIgA is directly related to local immunity. SIgA also functions as a protector by (I) preventing pathogenic microorganisms from adhering to the mucosal epithelial cells; (II) neutralizing intracellular pathogens by fusing with lysosome; (III) eliminating pathogens on the mucosal lamina propria; and (IV) mediating antibody-dependent cellular cytotoxicity (ADCC). In addition to SIgA, B cells in local mucosal tissues can also produce $\operatorname{IgG}$ and $\operatorname{IgM}$ independently. IgG antibodies in the blood can also diffuse to the surface of the mucosa. IgG is a group of proteins with antibody biological activity synthesized and secreted by the plasma cells of the body. It is the most abundant immunoglobulin in the body, making up approximately $75-$ $80 \%$ of the antibodies in our blood. It is also the main type of antibody found in blood and extracellular fluid and is one of the major antibodies involved in the body's secondary immune response.

In addition to humoral immunity, mucosal immunity also produces mucosal cytotoxic $T$ lymphocyte responses (CTLs) $(14,15)$. Mature T lymphocytes express CD4+ and CD8+ cells. CD4+ cells, including subsets of Th1, Th2, Th17, and Treg and acting as the primary cells involved in tumor immunity, assist and boost other immune cells in killing tumor cells by secreting cytokines. Th1 cells are involved in cell-mediated immunity through the expression of IL-2, IL12 , IFN- $\gamma$, and TNF- $\alpha$. Th2 cells are involved in humoral immunity through the expression of IL-4, IL-5, IL-6, and IL-10. Normally, Th1 cells and Th2 cells keep in a state of dynamic equilibrium in the body and the shift from Th1 to Th2 is closely associated with tumors.

HPV infections are unclear with no apparent early signs and symptoms. Upon the infection with HPV, the body will activate the autoimmune response to eliminate the HPV. Previous studies suggest that innate immunity will be activated within 96 hours after the HPV invasion, serving as the first barrier against HPV infection and eliminating more than $90 \%$ of pathogens (16). Adaptive immunity will also be activated after 96 hours. Viral antigen can induce specific immune responses, including cell-mediated immune responses and humoral immunity. HPV infections are restricted to the epidermal layer of skin mucosa with little or no activity in the blood, therefore, cell-mediated immunity plays a major role in the elimination of the virus. There is a well-established immune system in the body that protects the body from a microorganism, including viruses and infections. But immune evasion is detected in HPVpersistent infection. HPV viral antigens are difficult to contact with the antigen-presenting cells in the infected area and can be identified, hampering the activation of the innate immune response and adaptive immunity and resulting in the persistent proliferation of HPV in the infected area.

In the present study, the levels of SIgA, IgG, and IL-10 were positively correlated with the degree of cervical lesions and IL-2/IL-10 was negatively correlated with the degree in all patients before treatment. The levels of SIgA, IgG, and IL-10 were positively correlated with the degree of cervical lesions and IL-2/IL-10 was negatively correlated with the degree in HPV16 and HPV18-infected patients before treatment. In clinical diagnosis cases of rheumatoid arthritis, the combined detection complements the $\mathrm{C} 3$ levels and the C4 levels contribute to the accuracy of the diagnosis. IgM, IgA, and IgG are significant in the diagnosis of pediatric mycoplasma pneumonia infection. Pediatric mycoplasma pneumonia infection is attributable to immunodeficiency and immune dysfunction. It is recommended to detect the immunoglobulin levels to improve the diagnosis of mycoplasma pneumonia infection in children and provide guidance on clinical medication. The SIgA in the mucosal immune system is the first involved in the virus elimination upon HPV invasion. When the viral load is beyond the immunity limit of the mucosal local SIgA, the cell-mediated immune response will be activated for the further elimination of HPV. As the disease progresses, the local humoral immunity at the mucosal surface will be less effective and the cell-mediated immunity will take over. Thus, humoral immune factors, such as SIgA and IgG in patients with LSIL are expressed at a lower level when compared to the control group, indicating that the immune system of the body is activated to resist the disease progression. In patients with HSIL, both humoral immune factors, such as SIgA and IgG and cellular immune factors, such as IL-2 and IL-10 are expressed at a high level and the IL-2/IL-10 ratio is balanced; the immune system of the body is out of balance and the disease is progressive.

Pathological diagnoses are the golden standard for 
cervical lesions, but such diagnosis is invasive. Cytodiagnosis functions as the preliminary screening of cervical lesions, although it has certain limitations. Clinically, it is recommended to determine the levels of the vaginal local immune factors to make up for the limitation of cellular diagnosis to promote a noninvasive diagnosis. However, the medical reference value of the relevant immune factors needs to be verified in larger trials. Current HPV-DNA detection techniques can identify the HPV infection in the cervical cells of the host but cannot determine the active state after the infection has been contracted (16-20). The detection of HPV16/18E6/E7 protein cannot only identify the active state of HPV in the body but can also make up for the deficiency of available DNA and E6/E7mRNA detection technology. Therefore, SIgA, IgG, IL-2, IL-10, and the IL-2/IL-10 ratio is expected to be specific markers in cervical lesions and predictive factors for the disease outcome. Noninvasive diagnoses of cervical lesions can be achieved through the monitoring of immunological indexes.

TCT and HPV are the common detection methods used during the follow-ups of patients treated for cervical lesions and those with persistent cytological abnormality or HPV infection may undergo additional invasive examination (cervical biopsy). There is no noninvasive and highly sensitive clinical test method for monitoring the prognosis of cervical lesions yet. In the present study, all patients with cervical lesions underwent a 1-year follow-up after treatment and were subject to the monitoring of local vaginal immunity before treatment and 3, 6, and 12 months after treatment. The results showed that in the LSIL group, the negative conversion rate of HPV was positively correlated with the extension of the follow-up duration and the IL-10 level was positively correlated with the follow-up duration in the LSIL patients. IgG and IL-2 were expressed at a low level and IL-2/IL-10 was expressed at a high level 6 months after treatment. It was, therefore, concluded that in the early treatment of LSIL, SIgA in the mucosal immune system played a major role in the early elimination of HPV and the treatment of the disease but SIgA functioned in a short period and lost its role in the further treatment of the disease. After treatment, the immune system of the patients with LSIL repaired with time and IgG, IL2 , and IL-10 were highly expressed. At 6 months after treatment, however, all immune factors were expressed at a low level, indicating that the patients' immunity was recovering. All immune factors were expressed at higher levels with the extension of the follow-up duration and the patients' immune system gradually returned to normal.
Thus, HPV16 and HPV18 were eliminated the most at the 12 th month after treatment. So, we concluded that local vaginal immunity is directly correlated with HPV elimination. Clinically, it is feasible to determine the levels of local vaginal immune factors at different time points after treatment to identify the state of the immune function of the patients with cervical lesions to determine the prognosis of cervical lesions and HPV outcome: is the disease controlled? Or, will the disease progress? When the determined SIgA is lower and IgG higher than that before treatment, cellular immune factors are highly expressed and the IL-2/IL-10 ratio is balanced; the immune system of the body is considered stable and the cervical diseases are healing. On the contrary, high expression of the SIgA and low expressions of IgG, IL-2, and IL-10 and an imbalance of the IL-2/IL-10 ratio indicate an activated state of the immune system of the body to prevent the progression of the disease.

The levels of $\operatorname{IgA}, \operatorname{IgG}$, and $\operatorname{Ig} M$ in patients with leukemia were significantly lower than those in the control group and after treatment while the IgA, IgG, and IgM levels in the improved group were significantly higher than those before therapy, indicating a significant improvement of immunoglobulin levels. Thus, the detection of these indexes is helpful for disease monitoring and prognosis evaluation. In the process of the clinical treatment of patients with advanced gastric cancer, the application of laparoscopy-assisted D2 radical gastrectomy for gastric cancer patients can significantly improve the content of cytokines and help to prolong the patients' survival time. Such a technique should, therefore, be widely promoted. Immunoglobulin (IgG) heavy/light chain (HLC) is effective in the detection of minimal residual disease in patients with IgG type multiple myeloma (MM) after chemotherapy and indicative in the assessment of prognosis. In the present study, the negative conversion rate of HPV was positively correlated with the extension of the follow-up duration and the SIgA and IgG levels were negatively correlated with the extension of the follow-up duration in HSIL patients. IL-2 and IL-10 were expressed at a low level and IL-2/IL-10 was expressed at the lowest level 6 months after treatment, indicating that the humoral immunity lost its role in the late treatment of HSIL. IL-2 and IL-10 were at high levels before treatment and increased with the extension of the follow-ups and cell-mediated immunity plays a major role in curing the disease. However, these indexes were expressed at a low level 6 months after treatment and the ratio of both factors reached the trough value to a balanced 
state after treatment, suggesting that the immune system starts to recover 6 months after treatment. The immune system of patients with LSIL or HSIL returns to normal at 6 months after treatment, at which time the levels of local immune factors can be increased to enhance local immunity to ensure the highest HPV elimination at the 12 th month after treatment.

It is feasible to determine the levels of local vaginal immune factors of patients with cervical disease at different time points after treatment to determine the prognosis and guide the treatment of the disease. If the local immune factors IgG, IL-2, and IL-10 increase, the IL-2/IL-10 ratio is balanced, and the immune system is activated; it can be considered that the cervical disease is in the LSIL level and under treatment. If humoral immune factors are expressed at a low level, IL-2 and IL-10 are expressed at an extremely high level, and the IL-2/IL-10 ratio is far imbalanced, it can be considered that the immune system in the patients with HSIL is recovering and that HSIL is under treatment. Therefore, local immunity enhancement is recommended for curing HSIL.

In this study, we assessed the characteristics of local vaginal immunity of patients with cervical lesions to gain insight into the local vaginal immune state from the perspective of the function of local vaginal immunity. After treatment, the recovery of the immune system and the elimination of HPV16 and HPV18 in patients with cervical lesions were time-dependent. As previously mentioned, the immune system starts to recover at 6 months after treatment. There is no available clinical management in the reexamination of HPV in patients with cervical diseases after treatment yet. So, it is acceptable to monitor the immune indexes of these patients from the 6th month after treatment, combined with the purposeful enhancement of the local immune status. But early intervention is not recommended because, with the extension of the followup, the autoimmune system returns to normal and can eliminate the HPV infection. After 12 months of treatment, the immune system recovers to normal and the assessment of the efficacy can be initiated. If HPV has not been eliminated to an ideal level at 12 months after treatment, the disease may persist or progress, so clinical intervention is recommended.

Limitations. Firstly, this trial was not a randomized controlled trial. Secondly, this study was only a singlecenter trial and the sample size was limited. Thirdly, the clinical follow-up was short and it was necessary to observe the clinical long-term prognosis.

\section{Conclusions}

Firstly, the recovery after the treatment of HPV infection is time-dependent and early intervention is not recommended. Secondly, the immune system of patients with CIN starts to recover in the 6th month after treatment, at which time the immune indexes can be monitored to boost local immunity and early intervention is not recommended. Thirdly, the immune system is approximately in a normal state at 12 months after treatment, at which time the efficacy can be evaluated, and clinical intervention can be initiated if necessary. Lastly, the level of local immune factors in the vagina can be monitored to determine the progression and prognosis of cervical lesions.

\section{Acknowledgments}

We would like to acknowledge the hard and dedicated work of all the staff that implemented the intervention and evaluation components of the study.

Funding: National Natural Science Foundation of China (81260095) and Special Program of Health Industry, NHFPC (201402010).

\section{Footnote}

Data Sharing Statement: Available at http://dx.doi. org/10.21037/tcr-19-2955

Conflicts of Interest: All authors have completed the ICMJE uniform disclosure form (available at http://dx.doi. org/10.21037/tcr-19-2955). The authors have no conflicts of interest to declare.

Ethical Statement: The authors are accountable for all aspects of the work in ensuring that questions related to the accuracy or integrity of any part of the work are appropriately investigated and resolved. The study was conducted in accordance with the Declaration of Helsinki (as revised in 2013). The study was approved by institution ethics board of Affiliated Hospital of Inner Mongolia Medical University (No.: 2019029) and informed consent was taken from all the patients.

Open Access Statement: This is an Open Access article distributed in accordance with the Creative Commons Attribution-NonCommercial-NoDerivs 4.0 International 
License (CC BY-NC-ND 4.0), which permits the noncommercial replication and distribution of the article with the strict proviso that no changes or edits are made and the original work is properly cited (including links to both the formal publication through the relevant DOI and the license). See: https://creativecommons.org/licenses/by-nc-nd/4.0/.

\section{References}

1. Hu SY, Remila R, Zhang Q, et al. Progression and regression of women with biopsy-confirmed normal cervical epithelium or cervical intraepithelial neoplasia grade 1: a 15-year population-based cohort study in China. Lancet 2017;390:S99.

2. Luttjeboer J, Daemen TC. Threshold cost-effectiveness analysis for a therapeutic vaccine against HPV16/18-positive cervical intraepithelial neoplasia in the Netherlands. Vaccine 2016;34:6381-7.

3. He Y, Zhao Q, Geng YN. Clinical analysis of cervical intraepithelial neoplasia with vaginal intraepithelial neoplasia. Medicine 2017;96:e6700.

4. Byun JM, Jeong DH, Kim YN, et al. Persistent HPV16 infection leads to recurrence of high-grade cervical intraepithelial neoplasia. Medicine 2018;97:e13606.

5. Cordeiro JC, Da Silva JS, Roxo VS, et al. A pilot study on Hla-G locus control region haplotypes and cervical intraepithelial neoplasias. Hum Immunol 2017;78:281-6.

6. Lababidi S, Yan D, Cook IC, et al. Progression and recurrence patterns of vulvar intraepithelial neoplasia. Gynecol Oncol 2018;149:628.

7. Vasiljević N, Carter PD, Reuter C, et al. Role of quantitative p16(INK4A) mRNA assay and digital reading of p16(INK4A) immunostained sections in diagnosis of cervical intraepithelial neoplasia. Int J Cancer 2017;141:829-36.

8. Leng CY, Low H, Chua L, et al. Human papillomavirus 16 (HPV16) and HPV52 E6-specific immunity in HIVinfected adults on combination antiretroviral therapy. HIV Med 2017;18:321-31.

9. Shannon B, Yi TJ, Perusini S, et al. Association of HPV infection and clearance with cervicovaginal immunology and the vaginal microbiota. Mucosal Immunol 2017;10:1310-9.

10. Łaniewski P, Barnes D, Goulder A, et al. Linking cervicovaginal immune signatures, HPV and microbiota composition in cervical carcinogenesis in non-Hispanic and Hispanic women. Sci Rep 2018;8:7593.

11. Date Y, Ebisawa M, Fukuda S, et al. NALT M cells are important for immune induction for the common mucosal immune system. Int Immunol 2017;29:471-8.

12. Powell N, Walker MM, Talley NJ. The mucosal immune system: master regulator of bidirectional gutbrain communications. Nat Rev Gastroenterol Hepatol 2017;14:143-59.

13. Gonçalves P, Araújo JR, Di Santo JP. A Cross-Talk Between Microbiota-Derived Short-Chain Fatty Acids and the Host Mucosal Immune System Regulates Intestinal Homeostasis and Inflammatory Bowel Disease. Inflamm Bowel Dis 2018;24:558-72.

14. Brandtzaeg P. Mucosal Immunity: Induction, Dissemination, and Effector Functions. Scand J Immunol 2009;70:505-15.

15. Müller CA, Autenrieth IB, Peschel A. Intestinal epithelial barrier and mucosal immunity. Cell Mol Life Sci 2005;62:1322.

16. Massa S, Paolini F, Curzio G, et al. A plant protein signal sequence improved humoral immune response to HPV prophylactic and therapeutic DNA vaccines. Hum Vaccin Immunother 2017;13:271-82.

17. Tsakogiannis D, Gartzonika C, Levidiotou-Stefanou S, et al. Molecular approaches for HPV genotyping and HPVDNA physical status. Expert Rev Mol Med 2017;19:e1.

18. Tawe L, Grover S, Narasimhamurthy M, et al. Molecular detection of human papillomavirus (HPV) in highly fragmented DNA from cervical cancer biopsies using double-nested PCR. MethodsX 2018;5:569-78.

19. Obiri-Yeboah D, Adu-Sarkodie Y, Djigma F, et al. Options in human papillomavirus (HPV) detection for cervical cancer screening: comparison between full genotyping and a rapid qualitative HPV-DNA assay in Ghana. Gynecol Oncol Res Pract 2017;4:5.

20. Surriabre P, Allende G, Prado M, et al. Self-sampling for human papillomavirus DNA detection: a preliminary study of compliance and feasibility in BOLIVIA. BMC Womens Health 2017;17:135.

Cite this article as: $\mathrm{Mu} \mathrm{L}$, Miao JR, Song JH. The correlation of HPV16 and HPV18 with local vaginal immunity after the treatment of cervical intraepithelial neoplasia. Transl Cancer Res 2020;9(7):4212-4223. doi: 10.21037/tcr-19-2955 